\title{
Miradas multimedia sobre resiliencia y educación: innovación educomunicativa para la resiliencia de la infancia en riesgo social
}

María-Teresa Rascón-Gómez, Florencio Cabello Fernández-Delgado y Alejandro AlvaradoJódar

\section{RESUMEN}

El Proyecto de Innovación Educativa "Miradas multimedia sobre resiliencia y educación”, desarrollado en el curso académico 2015/2016 en la Universidad de Málaga (España)', surge en el contexto de la crisis económica que padece nuestro país desde 2008 y que se ceba especialmente con la infancia, alcanzando unas cotas insostenibles de riesgo de pobreza y exclusión social en esta población. Esta iniciativa educomunicativa, de inequívoco acento dialéctico, dialógico y democratizador, pone en relación a docentes del ámbito de la educación, comunicación y psicología, a alumnos de los grados de Educación social, Publicidad y Relaciones Públicas y Comunicación audiovisual y a entidades sociales con el fin de elaborar una serie de videos multimedia (spot, corporativo y web) y un cortometraje documental, para dar a conocer y reflexionar sobre los procesos de exclusión social y resiliencia de la infancia malagueña.

Palabras clave: innovación educativa, materiales multimedia, resiliencia, infancia, pobreza, España. la Universidad de Málaga, España. Temas de investigación: educación inclusiva, educación intercultural, tecnología y educación, género y formación de profesores.

\section{Florencio Cabello-Fernández-Delgado}

\section{Alejandro Alvarado-Jódar}

\footnotetext{
${ }^{1}$ Este proyecto forma parte del PIE 15-156 "Miradas multimedia sobre resiliencia educación: Narración audiovisual para la promoción de la infancia en riesgo de exclusión social”, financiado por el Vicerrectorado de Personal Docente e Investigador de la Universidad de Málaga en el marco de su convocatoria de Proyectos de Innovación Educativa (PIE) (2015-2017). A su vez, dicho PIE tiene su matriz en el Proyecto Investigación de Excelencia SEJ 13-66 "Trabajo en red y Atención Socioeducativa para la promoción de la Resiliencia de la Infancia en Riesgo Social", financiado por la Consejería de Economía y Conocimiento de la Junta de Andalucía (2013-2017). Para más información sobre la actividad del proyecto, véase: https://www.facebook.com/rieduc.
} 
Olhares multimídia sobre resiliência e educação: Inovação educomunicativa para a resiliência da infância em risco social

\title{
RESUMO
}

O Projeto de Inovação Educativa "Olhares multimídia sobre resiliência e educação", desenvolvido no período académico 2015/2016 na Universidade de Málaga (Espanha), surge no contexto da crise económica que padece nosso país desde 2008 e que se recai especialmente na infância, alcançando cotas insustentáveis de risco de pobreza e exclusão social nesta população. Esta iniciativa educomunicativa, de inequívoco acento dialético, dialógico e democratizador, põe em relação a docentes do âmbito da educação, comunicação e psicologia, a alunos dos graus de Educação social, Publicidade e relações públicas e Comunicação audiovisual e a entidades sociais com o fim de elaborar uma série de vídeos multimídia (spot, corporativo e web) e um documentário curta-metragem, para revelar e refletir sobre os processos de exclusão social e resiliência da infância malaguenha.

Palavras chave: inovação educativa, materiais multimídia, resiliência, infância, pobreza, Espanha.

\section{Multimedia Views on Resilience and Education: Educommunicative Innovation for the Resilience of Children at Social Risk}

\begin{abstract}
The Educational Innovation Project "Multimedia views on resilience and education", developed in the academic year 2015/2016 at the University of Malaga (Spain), arises in the context of the economic crisis that our country has been suffering since 2008 and which is especially affecting children, reaching unsustainable levels of risk of poverty and social exclusion in this population. This educational and communicative initiative, with an unequivocal dialectic, dialogical and democratizing accent, brings together teachers from the fields of education, communication and psychology, students from the grades of Social Education, Advertising and Public Relations and Audiovisual Communication, together with social entities as well with the aim of producing a series of multimedia videos (spot, corporate and web) and a short documentary film in order to make known and reflect on the processes of social exclusion and resilience of Malaga's children.
\end{abstract}

Key words: educational innovation, multimedia materials, resilience, childhood, poverty, Spain. 


\section{Introducción}

El colapso financiero desencadenado en 2008 ha tenido un reflejo dramático en el bienestar social en España, situándola como un país en acelerado empobrecimiento. Según la Fundación de Fomento de Estudios Sociales y Sociología Aplicada (FOESSA, 2016a), la población española es una de las más afectadas por la crisis económica, con un aumento tanto de la tasa de pobreza como de la desigualdad en los últimos años que apenas tiene parangón en la Unión Europea (UE). Además, estos indicadores tan preocupantes lo son aún más por el modo también desigual en que afectan a los distintos territorios de España, situándose Andalucía como una de las comunidades autónomas que persistentemente ocupan los últimos puestos en estas mediciones. En consonancia con ello, el porcentaje de población en riesgo de pobreza o exclusión social se sitúa en 2014 en $28.6 \%$ a escala nacional, elevándose en Andalucía hasta un 43.2\%, lo cual sitúa esta región como líder absoluta a este respecto (Instituto Nacional de Estadística (INE), 2016a). Para completar el cuadro, el INE (2016b) cifra la tasa de desempleo en España en un 21\% (46.5\% para menores de 25 años) y en Andalucía en un $29.7 \%$ (60.1\% para menores de 25 años).

Esta dramática tendencia en los indicadores de pobreza y desigualdad en España se ceba especialmente con la infancia, cuyas tasas de pobreza eran ya elevadas antes de la expansión de las políticas de austeridad en Europa pero cuyo riesgo de pobreza y exclusión social alcanza sus mayores cotas recientemente, elevándose hasta el 35.3\% en 2015 (INE, 2016a). Estamos, pues, ante una población especialmente vulnerable sobre la que se cierne la doble amenaza de la "transmisión intergeneracional de la pobreza" (FOESSA, 2016b) y de la cronificación de su exclusión frente a las mayores probabilidades de escapar de ella de la población adulta.

Frente a estas dinámicas de desposesión y desigualdad agudas, consideramos imprescindible desplegar enfoques que acometan intervenciones integrales acordes con la multifactorialidad que caracteriza las situaciones de pobreza y exclusión social y que apuesten por el trabajo en red entre las instituciones públicas y las entidades del tercer sector (Organismos Gubernamentales (ONG), asociaciones, fundaciones sin ánimo de lucro...). Para ello estimamos relevante la identificación, el estudio y la ulterior difusión de modelos de intervención con infancia en riesgo social que conjuguen compromiso social, rigor en el diseño y evaluación y capacidad de innovación y experimentación. En este proceso resulta crucial que los sujetos y grupos afectados mantengan un protagonismo destacado que revele sus factores de protección personales y socioculturales con vistas a reconocer y estimular sus potencialidades de superación, (auto)transformación y mejora. En este sentido, nos interesa la noción de resiliencia, entendida como "un proceso dinámico, constructivo, de origen interactivo, sociocultural que conduce a la optimización de los recursos humanos y permite sobreponerse a las situaciones adversas" (Kotliarenco y Cáceres, 2011: 1).

He aquí las claves de las que surge el Proyecto de Innovación Educativa (PIE) de la Universidad de Málaga (UMA) "Miradas multimedia sobre resiliencia y educación" cuyo diseño, desarrollo y resultados durante el curso 2015-2016 presentamos y discutimos a continuación. Se trata de una iniciativa de un grupo de 10 docentes de Educación, Comunicación y Psicología que deseamos propiciar que los estudiantes de Educación y Comunicación de la UMA conozcan y reflexionen sobre los procesos de exclusión social y de resiliencia de la infancia malagueña para ulteriormente elaborar sus propias narraciones multimedia al respecto. Nuestra concepción de este PIE se enmarca en el campo de la "educomunicación", término que preferimos a otros asociados como "alfabetización mediática" (Kendall y McDougall, 2012; Gutiérrez y Tyner, 2012; Area-Moreira y Ribeiro-Pessoa, 2012), por el inequívoco acento dialéctico, dialógico y democratizador que aquel pone en la imbricación entre educación y comunicación. Así, 
mientras que determinadas concepciones de la media literacy adoptan un enfoque economicista orientado a la eficaz instrucción en unos medios supuestamente neutrales (Aparici, 2010: 11) o abrazan sin más un paradigma de "sonambulismo tecnológico y amnesia educativa" (Cabello y Rascón, 2015), y mientras que términos como "educación para la comunicación" y "comunicación para la educación" entrañan el riesgo reduccionista de "escolarizar la comunicación o tecnificar la educación" (Huergo, citado en Oliveira, 2010: 130), la educomunicación remite explícitamente a la tradición de la pedagogía de la liberación y la pedagogía de la comunicación auspiciada desde los setenta del siglo pasado por Paulo Freire, Mario Kaplún, Daniel Prieto o Francisco Gutiérrez (Aparici, 2010; Abellán y Mayugo, 2008).

De este modo, frente a modelos de educación/ comunicación vinculados a lo que Kaplún (citado en Barranquero, 2009) denomina "pedagogía transmisora" o "pedagogía persuasivo-conductista", encontramos la defensa de una "pedagogía problematizadora" como impulsora de la reflexión y acción autónomas de los educandos, organizados en un marco de interacción "que apuesta por el grupo y su capacidad autogestora" (Kaplún, 2010: 55). Dicha pedagogía problematizadora se halla en última instancia inspirada por la concepción comunicativa propuesta por Paulo Freire a comienzos de los setenta y que Barranquero (2007: 117) sintetiza así: "Según Freire, la comunicación vendría a ser sinónimo de diálogo [...] La auténtica comunicación es [...] según este modelo, dialéctica $[\mathrm{y}]$ de este modo genera ‘concientización' en el doble sentido político-pedagógico freireano, como conocimiento (o descubrimiento de la razón de las cosas) y como conciencia (de sí, del otro, de la realidad)".

A partir de estos fundamentos, llegamos en nuestros días a una consolidación de un campo mixto cuyas aspiraciones recapitula García (2010: 151152) afirmando que: "la educomunicación ofrece los instrumentos para comprender la producción social de comunicación, saber valorar cómo funcionan las estructuras de poder, cuáles son las técnicas y los elementos expresivos que los medios manejan y poder apreciar los mensajes con suficiente distanciamiento crítico, minimizando los riesgos de manipulación".

Para completar este sucinto repaso conceptual concluimos mencionando la riqueza de matices que añade Barranquero (2007) en su genealogía y definición de la "educomunicación para el cambio social". De este modo, partiendo de un recorrido histórico que bebe de Freire pero también de la contemporánea emergencia en Latinoamérica de una escuela comunicativa crítica que forjaría el paradigma de la "comunicación para el cambio social" (Gray-Felder y Deane, 1999), Barranquero (2007) proporciona siete claves para planificar iniciativas de este tipo que dibujan las coordenadas básicas en las que intentamos que se mueva nuestro PIE.

Lo primero de todo es la primacía del "proceso de transformación colectiva" por encima de los productos mediáticos que emanen de él. En segundo lugar, destaca la importancia de que los actores implicados en tal proceso acaben apropiándose del mismo, haciéndolo suyo. Junto a ello, Barranquero (2007) hace hincapié en la necesaria "pertinencia cultural" de las acciones que se diseñen, con el fin de atender a las particularidades culturales y lingüísticas de cada comunidad. Las características cuarta y quinta van emparentadas, pues si bien se enfatiza el interés de intervenciones apegadas al terreno que generen una participación distribuida en lo local, no se pierde de vista la necesidad de un trabajo en red que permita vincular entre sí experiencias similares desplegadas en contextos diversos, algo ya defendido por Kaplún (2010: 57). años atrás: "Así como [...] un grupo es mucho más que la suma de sus miembros, también una red intergrupal es mucho más que la suma de los grupos que la integran [...] Sería, por otra parte, en esta comunicación intergrupal a distancia donde el empleo de medios encontraría su plena justificación y su legitimación". 
En cuanto a la selección de medios tecnológicos, se apuesta siempre por su adecuación al contexto y se rechaza que acaben convertidos en fines en sí mismos. Para finalizar, Barranquero (2007) subraya que es imprescindible que las acciones contemplen metas a medio y largo plazo que posibiliten una auténtica apropiación de los procesos y una transformación duradera de las condiciones de vida de las comunidades.

\section{Diseño metodológico}

A partir de las premisas educomunicativas arriba señaladas, y con referentes de carácter nacional e internacional, como el de la agencia de comunicación solidaria Comsolidar creada por el profesor Marcial García en la Universidad de Málaga (Olmedo y Ruiz, 2013), o la experiencia de implementación de una propuesta comunicativa desarrollada en la Universidad Autónoma de Chihuahua por los profesores Ávila y Cortés (2016), nuestro Proyecto de Innovación Educativa (PIE) plantea para los cursos 20152016 y 2016-2017 una experiencia de aprendizaje cooperativo basado en proyectos a caballo entre la educación social y la comunicación audiovisual. Respecto al aprendizaje cooperativo, nos remitimos a la clásica definición del mismo como "el uso en educación de grupos pequeños en los que los alumnos trabajan juntos para mejorar su propio aprendizaje y el de los demás" (Johnson y Johnson, 1999: 20). Por lo que se refiere al proyecto propuesto a nuestros estudiantes para que integren en torno a ellos los aprendizajes y competencias adquiridos durante el curso, posee una doble dimensión, a saber, de aprendizaje-servicio (APS) y de realización multimedia.

Dicha conjunción de dimensiones educomunicativas obedece a los distintos grados a los que pertenecen los 124 estudiantes implicados en el PIE (60 de Educación Social, 60 de Publicidad y Relaciones Públicas y 4 de Comunicación Audiovisual) y, más específicamente, a las cuatro asignaturas involucradas ("La educación social ante la exclusión y la marginación" e "Infancia y juventud en riesgo social", materias obligatorias que cursan en el primer y segundo cuatrimestre los alumnos de $2^{\circ}$ de Educación Social; "Realización de vídeos multimedia", optativa de segundo cuatrimestre de $3^{\circ}$ de Publicidad y RRPP; y "Realización de Documental", optativa de segundo cuatrimestre de $4^{\circ}$ de Comunicación Audiovisual).

Los objetivos generales de nuestro proyecto son, por un lado, contribuir a la aproximación crítica del alumnado universitario a los procesos de exclusión social de la infancia malagueña y a las iniciativas socioeducativas que promueven su resiliencia, incluyendo el modo en que vienen mediados por sus representaciones audiovisuales; y por otro, divulgar dichos procesos e iniciativas entre la comunidad universitaria y la ciudadanía en general, partiendo siempre del entorno más próximo pero con vocación de trascender a otras universidades y localidades. Más específicamente, aspiramos a que nuestro alumnado identifique en su entorno y difunda buenas prácticas socioeducativas de trabajo en red en pro de la resiliencia de la infancia en riesgo social; a que adquiera un compromiso ético con sus sujetos de estudio/intervención, con especial acento en el análisis crítico de la construcción mediática de las figuras y los entornos de exclusión social, y finalmente, a que devuelva a las comunidades el material elaborado, además de propiciar su difusión amplia mediante su archivo en el repositorio de conocimiento abierto de la UMA.

A partir de esta apuesta por "la dimensión comunitaria de la educación en comunicación” (Abellán y Mayugo, 2008), nuestro PIE contempla dos fases de desarrollo. La primera, centrada en el primer cuatrimestre, propone que los estudiantes de Educación Social se aproximen a comunidades del entorno de Málaga donde existan problemas de exclusión social e identifiquen las demandas de intervenciones socioeducativas para promover la resiliencia de la infancia o juventud en riesgo. A partir de aquí, los futuros 
educadores sociales han de diseñar en colaboración con dichas comunidades un proyecto de aprendizajeservicio (APS) que responda a sus necesidades y deseos. Si bien esta primera fase posee pleno sentido y validez en sí misma, en nuestro PIE se contempla asimismo como una oportunidad de practicar la "prealimentación" o "feed-forward" que Kaplún propuso en los setenta: "Si se desea comenzar un real proceso de comunicación, el primer paso debiera consistir en poner al destinatario, no sólo al final del esquema, sino también al principio, originando los mensajes, inspirándolos, como fuente de pre-alimentación [...] La verdadera comunicación no comienza hablando sino escuchando" (Kaplún, citado en Barranquero, 2009: 5).

La segunda fase comienza en el segundo cuatrimestre y supone ya la creación de grupos interdisciplinares de estudiantes (y sus docentes) de Educación Social, Publicidad y RRPP y Comunicación Audiovisual que sumen a las competencias educativas las de realización audiovisual con vistas a la producción y realización de narraciones multimedia sobre los APS. Más concretamente, se les encargan tres piezas (un spot publicitario de medio minuto, un video corporativo de tres minutos y un teaser orientado a la difusión web), excepto en el caso de los estudiantes de Comunicación Audiovisual, orientados a realizar un corto documental de unos diez minutos.

En la formación de los 12 grupos mixtos (compuestos por entre tres y cinco estudiantes de Educación y otros tantos de Comunicación) que emprendieron la segunda fase del PIE tuvimos en cuenta los cinco elementos esenciales de las iniciativas cooperativas fructíferas que contemplan Johnson y Johnson (1999):

a) En primer lugar, la interdependencia positiva de objetivos, la cual "se da cuando se establece un objetivo mutuo o conjunto de manera tal que los individuos perciben que sólo si sus compañeros alcanzan sus objetivos podrán ellos mismos alcanzar los propios" (Johnson y Johnson, 1999: 53). En nuestro caso, la interdependencia venía dada por el hecho de que las obras audiovisuales de los estudiantes de Comunicación se pusieran al servicio de las comunidades con las que los alumnos de Educación Social desarrollaron sus APS, y a su vez que estos proyectos pudieran alcanzar una mayor repercusión pública mediante los videos realizados por los futuros comunicadores.

b) En cuanto a la interacción promotora cara a cara, entrañaba cierta dificultad en la medida en que los grupos de trabajo los componían estudiantes de distintos centros, circunstancia que se replicaba en el caso de los docentes implicados. Con el fin de propiciar el contacto fluido y cara a cara entre alumnado y profesorado de Educación y Comunicación, el PIE contempló la realización de tres eventos conjuntos de coordinación a los que se invitó a todos los participantes y que se desarrollarían al principio del cuatrimestre: uno para introducir el PIE, otro posterior para conformar y coordinar los grupos y organizar su seguimiento, y el tercero antes del final de las clases, para una revisión conjunta de los primeros resultados. Junto a ello, estimamos fundamental que cada docente involucrado en el PIE asumiera la tutorización personalizada de uno de los grupos mixtos para facilitar su coordinación y descargar de trabajo a los dos coordinadores de las asignaturas y al propio coordinador del PIE.

c) En nuestro caso, el fomento de la responsabilidad individual y personal no requirió un esfuerzo extraordinario en la medida en que en general los estudiantes demostraron desde el inicio un alto grado de compromiso con el trabajo, no sólo en su dimensión académica sino también social, para lo cual la fase de prealimentación aludida resultó clave.

d) Por lo que respecta al uso de habilidades interpersonales en grupos pequeños, se trata de una competencia transversal para todos los estudiantes y docentes involucrados, si bien en este PIE entrañaba un 
reto adicional por el hecho de unir a estudiantes de distintos grados sin experiencia previa de colaboración. En este punto, la tarea de los tutores asignados a cada grupo pequeño volvía a revelarse como clave para detectar los posibles conflictos y fomentar un ambiente cooperativo propicio.

e) Uno de nuestros mayores desafíos ha radicado en articular con la suficiente complejidad y coherencia el procesamiento grupal del proceso y los productos surgidos, definido por Johnson y Johnson (1999: 129) como "la reflexión sobre una actividad grupal para ver qué acciones de sus integrantes resultaron útiles y cuáles no lo fueron y para tomar decisiones sobre qué acciones se deben conservar y cuáles se deben cambiar".

Y es que junto a la dimensión grupal de la propia evaluación oficial de las obras del alumnado (en la medida en que los responsables de las materias de Educación y Comunicación la negociarían conjuntamente, asignando un valor equitativo a las competencias de una y otra área), nuestro PIE contemplaba una instancia de evaluación intragrupal (por la que los estudiantes de una y otra titulación evaluarían mutuamente el correcto desarrollo del proyecto educomunicativo) y otra externa (la de las comunidades y asociaciones involucradas, que ofrecían su retroalimentación final a través de una plantilla de evaluación que se les pedía rellenar). Con el fin de que estas diversas instancias pudieran combinarse de modo fructífero, hicimos mucho hincapié en la relevancia del tercer encuentro citado más arriba (que asumiría una función de pre-evaluación conjunta con la participación de estudiantes, tutores y coordinadores de las asignaturas y del PIE) y diseñamos para la época de exámenes un cuarto evento de proyección pública de las piezas audiovisuales.

A este evento, organizado en las instalaciones de la UMA, invitaríamos a todos los estudiantes y docentes implicados en el PIE, pero también a todos los colectivos, asociaciones y sujetos colaboradores de los
APS y de sus respectivos vídeos. Con ello queríamos propiciar una suerte de cine-fórum donde los estudiantes pudieran mostrar su trabajo a sus compañeros y profesores, a los protagonistas de su APS y también a otras entidades, para al final generar un coloquio a partir de estas múltiples voces. Por supuesto, junto a esta dimensión dialógica orientada a la devolución del trabajo, resultaba importante que esta proyección incorporara un componente lúdico, por lo que planeamos culminarla con una merienda comunitaria que cumpliera la recomendación de Johnson y Johnson (1999) de unir el procesamiento grupal con el "festejo" del trabajo compartido.

\section{Resultados}

En coherencia con la premisa educomunicativa según la cual el proceso de transformación colectiva ha de primar sobre los productos mediáticos, en esta sección expondremos los hitos principales del desarrollo del PIE antes de referirnos a las obras audiovisuales concretas que han resultado de dicho proceso.

Comenzamos destacando que al finalizar la primera fase del PIE encontramos que varios grupos de Educación Social no deseaban continuar en el segundo cuatrimestre con los APS desarrollados previamente, por lo que los profesores de Educación emprendieron contactos con diversas asociaciones malagueñas implicadas en procesos socioeducativos de resiliencia con infancia, logrando finalmente que todos los grupos de Educación que lo deseaban pudieran abordar un nuevo APS.

En este sentido, se reveló muy oportuna la organización de una presentación de tres experiencias socioeducativas de trabajo en red en Málaga. Celebrado en el Aula Magna de la Facultad de Educación, este evento reunió por primera vez a los 124 estudiantes y 10 docentes implicados en el PIE y les permitió aproximarse a entidades y vecinos de tres barrios humildes de Málaga (Carretera de Cádiz, La Corta y Los Asperones) donde se dan situaciones de exclusión social de la infancia. Además de que las 
asociaciones participantes acabarían incorporándose al PIE, este evento permitió que el alumnado visualizara un modelo del trabajo que se le encargaba con el estreno del video-reportaje institucional sobre el programa "Asperones Avanza"2 donde participaron dos de los docentes del PIE.

Tras este primer hito, los estudiantes tuvieron unas semanas para familiarizarse con la propuesta, pero de nuevo resultó esencial la celebración del segundo evento conjunto. Durante la celebración del mismo, los coordinadores del PIE realizaron una presentación detallada del mismo, presentaron a cada grupo mixto de estudiantes la persona que les tutorizaría y finalmente propiciaron que cada grupo se reuniera por separado con sus respectivos tutores y planificara las siguientes fases de trabajo.

Tras este segundo encuentro, surgieron algunos problemas de coordinación entre algunos grupos y las asociaciones con las que colaboraban, de modo que a mediados de cuatrimestre tuvimos que realizar los últimos reajustes en la formación de grupos, que quedaron de la siguiente manera:

- Nueve grupos mixtos de estudiantes de Educación Social y Publicidad y RRPP que colaboraban con las asociaciones Cruz Roja Málaga,Cruz Roja Fuengirola, Mesa de Trabajo Comunitario de La Corta, Asociación Trans, Casa Hogar La Colonia (FAISEM), Asociación de Vida Independiente, Casa Hogar La Buena Vida de La Palmilla, Granja El Pato (Fundación Espacio sin Exclusión) y Asociación Trencadís Romí de Los Asperones.

- Un grupo mixto de estudiantes de Educación Social y Comunicación Audiovisual que colaboraron con la comunidad socioeducativa de Los Asperones para la realización de un corto documental.
- Dos grupos de Educación Social que desarrollaron su APS sin recurso a los videos previstos y otros dos grupos de Publicidad y RRPP a los que propusimos enfocar sus obras audiovisuales a promocionar el propio PIE y el Proyecto de Investigación de Excelencia de donde surge.

Antes de la finalización de sus trabajos, todos estos grupos fueron convocados a una sesión de visionado conjunto de sus montajes provisionales. A esta sesión acudieron además todos los tutores y docentes implicados, y de este modo logramos una intensa sesión de procesamiento grupal donde los distintos aspectos de la evaluación eran discutidos públicamente con los estudiantes y, más allá, entre los propios docentes.

Tras esta sesión, se pidió a los estudiantes que culminaran sus montajes atendiendo a lo debatido, se les facilitaron los modelos de cesión de derechos y las plantillas de evaluación que deberían pasar a los protagonistas de sus videos al tiempo que se los hacían llegar y se les pidió que recabaran de éstos las plantillas rellenas y, más allá, el visto bueno y los permisos para difundirlas públicamente. Queremos reseñar que sólo una asociación denegó su permiso por discrepancias con el resultado final, mientras que el resto se mostró conforme con el trabajo y la mayoría emitió informes de evaluación muy positivos respecto de los APS y los videos, junto con valiosas sugerencias de mejora.

Completado este proceso, los docentes recibimos las piezas audiovisuales de los 12 grupos implicados en el $\mathrm{PIE}^{3}$ y con ellas organizamos una proyección conjunta en las instalaciones del Contenedor Cultural de la UMA. A esta sesión acudieron más de 100 personas, entre representantes de todos los colectivos y barrios colaboradores y estudiantes y docentes implicados. Tras una breve presentación y agradecimiento por parte de los organizadores, se pasó a un visionado de todos los videos autorizados, se siguió

\footnotetext{
${ }^{2}$ Véase: https://www.youtube.com/watch?v=nDxURMWcBk8.

3 Para una recopilación de los videos del PIE, véase: https://www.youtube.com/channel/UCD_VuhNj6oAhhjHLrjn6rnw/ videos?shelf_id=1\&sort=dd\&view $=0$.
} 
con un coloquio donde tomaron la palabra algunos protagonistas de los APS y finalmente se dio paso al festejo previsto en forma de merienda comunitaria. El satisfactorio resultado de este hito clave del procesamiento grupal facilitó mucho su culminación durante la sesión de evaluación conjunta celebrada una semana después con todos los docentes responsables de las asignaturas incluidas en el PIE.

Asimismo, es necesario resaltar la repercusión pública del PIE, que recibió un primer espaldarazo el 4 de junio cuando el principal diario de Málaga se hizo eco de la colaboración de la Asociación Trencadís Romí de Los Asperones con la Delegación de Igualdad de la UMA y difundió el video realizado por nuestras estudiantes sobre el proceso de construcción de un mural destinado a conmemorar el cuadragésimo aniversario de la Escuela Infantil de nuestra universidad (González, 2016). Junto a ello, los primeros resultados del proyecto se presentaron también en septiembre de 2016 en el Congreso Internacional de Pedagogía Social celebrado en Girona (España).

Antes de cerrar este apartado, nos gustaría mencionar la importancia que han tenido varios de los proyectos desarrollados por los alumnos de Comunicación Audiovisual y Educación Social en la promoción de la infancia en riesgo de exclusión social. Para ello, nos centraremos en los proyectos sobre la comunidad socioeducativa de Los Asperones y la Asociación Trencadís Romí, ambos desarrollados en ese barrio marginado de la capital de Málaga. La asociación Trencadís Romí es un grupo de niñas y mujeres de etnia gitana que se han unido para crear mosaicos con la técnica del "trencadís", basada en la creación de murales con trozos de azulejos rotos, la cual perfeccionan en talleres desarrollados por una educadora social y una artesana. El objetivo fundamental de esta iniciativa es crear redes resilientes comunitarias que permitan ampliar sus horizontes y proporcionarles una salida laboral. En este sentido, nuestro proyecto de innovación educativa ha permitido darle visibilidad a esta iniciativa mediante la elaboración de unos videos promocionales que se han presentado ya en diversos foros. Además, se han establecido redes de colaboración entre la Universidad de Málaga y el barrio de Los Asperones, mediante el encargo de diversos murales que se encuentran expuestos en dicha universidad, y que han servido para visibilizar el meritorio trabajo que realizan estas niñas y mujeres a partir de algo roto como son los azulejos. Pese a todas las dificultades, cuando escribimos esto, el proyecto "Trencadís" sigue activo en su intento por promover la inserción sociolaboral de estas jóvenes. Junto a ello, el proyecto les ha permitido conocer e interactuar con otras realidades ajenas al barrio, y mejorar su autoconcepto y autoestima.

Por otro lado, el corto documental desarrollado con la comunidad socioeducativa de Los Asperones ha servido para propiciar un mutuo acercamiento entre el barrio y la sociedad malagueña y su comunidad universitaria. De este modo los niños y adolescentes de Asperones han tenido la posibilidad de reunirse y establecer lazos con otros niños y jóvenes ajenos al barrio, y pertenecientes a otros estamentos sociales. En este sentido, varios de los participantes en el proyecto nos han confirmado que esa relación de cercanía ha permitido cuestionar estereotipos que la sociedad malagueña tiene sobre los habitantes del barrio. Cabe mencionarse como especialmente significativa del mutuo enriquecimiento referido la estrecha colaboración de un joven del barrio con un grupo de estudiantes que realizaban una suerte de video autopromocional sobre el grupo de investigación RIEDU (del que surge este PIE) ${ }_{1}^{4}$ colaboración de la que no sólo ha surgido una pieza destacable sino una ampliación de los vínculos de este joven con la parte estudiantil de la comunidad universitaria, pues hasta entonces sólo los tenía como participante/informante clave del citado RIEDU.

\footnotetext{
${ }^{4}$ Puede verse el spot sobre RIEDU en: https://bit.ly/2PZahel.
} 


\section{Discusión y conclusiones}

Este primer curso de experimentación con el Proyecto de Innovación Educomunicativa "Miradas multimedia sobre resiliencia y educación" ha suscitado en sus impulsores una serie de reflexiones, discusiones y dudas que queremos compartir aquí como un modo de ampliar aún más el alcance del procesamiento grupal de la experiencia.

En primer lugar, son numerosas las conclusiones halagüeñas que nos deparó el primer curso de desarrollo del PIE. Si el acento en la dimensión educomunicativa de nuestro proyecto entrañaba una apuesta por desencadenar procesos de transformación social, hemos de reconocer que tanto la evaluación continua en clase como la evaluación final con estudiantes (así como la evaluación de las asociaciones y colectivos implicados) nos han proporcionado pruebas de la transformación de los participantes, aunque sólo sea a escala personal. Así, no han faltado testimonios de estudiantes (sobre todo de Comunicación) que inicialmente confesaban su nerviosismo por acudir a determinados barrios marginados o centros asociativos de la ciudad y que han acabado bien integrados con sus habitantes y con las asociaciones que les atienden. Igualmente hemos percibido que determinados colectivos y asociaciones que manifestaban muchas reservas a ser grabados o a participar en la realización de anuncios o reportajes sobre su vida y su trabajo han modificado sus prejuicios al respecto y han percibido en nuestros estudiantes el compromiso ético con su situación y, más allá, aquella "empatía" que para Kaplún (citado en Barranquero, 2009: 5) es clave para la educomunicación y se define como "la capacidad de ponernos en la piel del otro, de sentir como él, de pensar como él, de 'sintonizar', de ponernos en su misma 'onda". En los mejores casos, hemos podido comprobar que las relaciones entabladas durante el curso se han prolongado una vez acabado éste, sobre todo en el caso de trabajo con jóvenes con edades similares a las de nuestros estudiantes universitarios.
Y esta dinámica de transformación personal no ha quedado reservada a estudiantes y vecinos o entidades, sino que los propios docentes hemos aprendido ampliamente de las experiencias y los problemas que han vivido nuestros estudiantes, y en ciertos casos hemos tenido que revisar estereotipos sobre la exclusión social en nuestra ciudad que difícilmente nos habríamos replanteado de otro modo. En definitiva, estimamos notable el cumplimiento de nuestro primer objetivo de propiciar un acercamiento a las situaciones de exclusión social de la infancia y juventud de Málaga y una implicación en los procesos socioeducativos que la combaten.

En cuanto a la divulgación de los productos audiovisuales generados en este primer curso, queremos subrayar que en general se ha realizado una acertada selección de las buenas prácticas socioeducativas de promoción de la resiliencia, con proyectos significativos que cubrían distintas zonas de la provincia de Málaga (y no sólo la capital) y que contaban con una trayectoria asentada y un horizonte de desarrollo que posibilita plantearse los objetivos a medio y largo plazo que defiende Barranquero (2007) para aspirar a un cambio prolongado y sostenible. Igualmente destacamos el excelente nivel general de las obras, tanto por lo que respecta a su contenido como a su creatividad y calidad estética y técnica, con algunos videos cuya factura es auténticamente profesional. Además, esta valoración ha sido compartida en buena medida por los colectivos involucrados, cuyas experiencias previas con la publicidad audiovisual de su labor eran muy limitadas y habitualmente amateur. Por lo demás, la difusión de los resultados del PIE ha quedado restringida por ahora a la comunidad universitaria malagueña y al entorno de las entidades y grupos con los que se ha colaborado, si bien noticias como la aparecida en el principal diario de Málaga o presentaciones como la desarrollada en el congreso de Girona apuntan ya en la dirección deseada de "evitar el localismo [e] impulsar la organización en red y vincular cada 
proyecto con otras experiencias similares a nivel local, regional o global" (Barranquero, 2007: 118).

En cuanto a los elementos de diseño directamente vinculados al aprendizaje cooperativo, destacamos una vez más la riqueza de un procesamiento grupal que ha acompañado el desarrollo del proyecto desde su arranque involucrando las perspectivas diversas de estudiantes, tutores, docentes y entidades en una colaboración mutuamente beneficiosa. Para ello, el papel de los tutores ha sido fundamental como mediadores entre los grupos de alumnos de cada grado, y entre éstos y las asociaciones, hasta el punto de que el único grupo que no recibió autorización para proyectar su video fue aquel cuyo tutor falló en sus funciones de coordinación, de modo que los responsables de la asignatura se encontraron el problema en un punto que no tenía fácil solución. Por otro lado, el problema sobrevenido de los grupos que se quedaron sin APS a mitad de curso nos llevó al hallazgo feliz de que dedicar un par de grupos a documentar y narrar el propio proceso del PIE y de sus proyectos asociados resulta un refuerzo muy interesante del procesamiento grupal, en la medida en que los distintos grupos sabían del trabajo y las impresiones de los demás a través de la realización de un vídeo específico al respecto.

También es reseñable que el profundo compromiso de los estudiantes con el PIE se ha traducido en un alto nivel de responsabilidad personal y grupal que incluso por momentos resultaba difícil de manejar por tutores y docentes y podía desembocar en conflictos intragrupales derivados de cierto perfeccionismo.

Continuando con el repaso a los elementos del aprendizaje cooperativo, consideramos que es preciso afinar aún más lo relativo a la interdependencia positiva de objetivos, pues si bien su formulación básica quedó clara para los estudiantes, nuestra explicación insuficiente de las competencias específicas y las distintas naturaleza y temporalidad de las tareas de educación social y realización audiovisual, generó confusiones y atribuciones erróneas de responsabilidades que dificultaron la coordinación. Por decirlo sintéticamente, necesitamos subrayar que los educadores sociales se basan en el tacto y los comunicadores en productos tangibles, y que aquéllos trabajan a fuego lento mientras que éstos persiguen los chispazos de la creatividad. En suma, asumimos la tensión entre la voluntad de transformación social y la vocación divulgativa de uno y otro campo, pero estamos convencidos de que una mejor labor pedagógica sobre educomunicación redundaría en una mejor percepción de los estudiantes del mutuo beneficio de su colaboración pese a las concesiones que lleva aparejadas.

Finalmente, estimamos que si bien la primera fase del PIE propició aquel momento de prealimentación que defiende Kaplún para la educomunicación, es imprescindible abundar en esta línea para evitar los malentendidos, recelos e incluso conflictos que han surgido con algunas asociaciones y han llevado incluso a que dos grupos tuvieran que desechar sus APS a mitad del segundo cuatrimestre. Para ello nos proponemos que el segundo curso de aplicación del PIE contemple una prealimentación no sólo vinculada a los APS sino también a las expectativas, necesidades y deseos respecto de las piezas multimedia que se proponen a los distintos colectivos. De ahí debería surgir un documento particular para cada entidad que, más allá del briefing genérico que se les da a todos los alumnos, contenga los compromisos y exigencias mutuos entre todas las partes de modo que, salvaguardando el derecho a veto que en última instancia corresponde a los destinatarios de los APS, garantice también que los estudiantes pueden desarrollar y difundir su trabajo al menos en el ámbito universitario, cumpliendo así sus expectativas respecto del PIE a la vez que intentan contribuir a la inclusión social de los sujetos con quienes desarrollan su labor educomunicativa. 


\section{Referencias}

Abellán, Gemma y Carme Mayugo (2008), "La dimensión comunitaria de la educación en comunicación", en Comunicar, núm. 31, Huelva, Ed. Grupo Comunicar, pp. 129-136.

Aparici, Roberto (2010), "Introducción: la educomunicación más allá del 2.0”, en Educomunicación: más allá del 2.0, Barcelona, Gedisa, pp. 9-23.

Area-Moreira, Manuel y María Teresa Ribeiro-Pessoa (2012), "De lo sólido a lo líquido: las nuevas alfabetizaciones ante los cambios culturales de la Web 2.0.”, en Comunicar, núm. 38, Huelva, Ed. Grupo Comunicar, pp. 13-20.

Ávila-Meléndez, Lilia Rosa y Jorge Abelardo CortésMontalvo (2016), “Comunic@tu servicio comunitario: una experiencia en la implementación de una propuesta educativa", en Revista Iberoamericana de Educación Superior, vol. VII, núm. 20, México, IISUE/Universia, pp. 60-77.

Barranquero, Alejandro (2009), Comunicación participativa y educación en medios. Implicaciones del concepto de prealimentación ("feed-forward») de Mario Kaplun, <http:// goo.gl/4QsCLg> [Consulta: enero de 2017].

Barranquero, Alejandro (2007), "Concepto, instrumentos y desafios de la edu-comunicación para el cambio social", en Comunicar, núm. 29, Huelva, Ed. Grupo Comunicar, pp. 115-120.

Cabello, Florencio y María Teresa Rascón (2015), "Aquellos que no recuerdan su pasado'... Sonambulismo y amnesia educativa: a vueltas con el fenómeno 'Ninguna diferencia significativa", en Redes. com, núm. 11, pp. 248-272.

Fundación de Fomento de Estudios Sociales y Sociología Aplicada (FOESSA) (2016a), Expulsión Socialy Recuperación Económica, Madrid, Cáritas Española, <http://goo.gl/ n9tL8Q> [Consulta: febrero de 2017].

FOESSA (2016b), La transmisión intergeneracional de la pobreza: factores, procesos y propuestas para la intervención, Madrid,
Cáritas Española Editores.

García, Agustín (2010), "Publicitar la educomunicación en la universidad del siglo XXI", en Educomunicación: más allá del 2.0, Barcelona, Gedisa, pp. 151-168.

González, Mari Ángeles (2016), "Romper azulejos para romper prejuicios", en SUR.es, 4 de Junio, <http://goo. gl/ELCOC> [Consulta: novienbre de 2016].

Gray-Felder, Denise y James Deane (1999), Communication for social change: a position paper and conference report, Nueva York, The Rockefeller Foundation.

Gutiérrez, Alfonso y Kathleen Tyner (2012), "Educación para los medios, alfabetización mediática y competencia digital", en Comunicar, núm. 38, Huelva, Ed. Grupo Comunicar, pp. 31-39.

Instituto Nacional de Estadística (INE) (2016a), Encuesta de Condiciones de Vida (ECV). Año 2015, <http://goo.gl/ w0gdQP> [Consulta: enero de 2017].

INE (2016b), Encuesta de Población Activa (EPA). Primer trimestre de 2016 (Nota informativa), <http://goo.gl/fQOlN2> [Consulta: enero de 2017].

Johnson, David y Rogers Johnson (1999), Aprender juntos y solos: aprendizaje cooperativo, competitivo e individualista, Buenos Aires, Aique.

Kaplún, Mario (2010), "Una pedagogía de la comunicación”, en Educomunicación: más allá del 2.0, Barcelona, Gedisa, pp. 41-61.

Kendall, Alex y Julian McDougall (2012), "Alfabetización mediática crítica en la postmodernidad", en Comunicar, núm. 38, Huelva, Ed. Grupo Comunicar, pp. 21-29.

Kotliarenco, María Angélica e Irma Cáceres (2011), Resiliencia y apego, Santiago de Chile, CEANIM, <http:/ / goo.gl/TH0WNi> [Consulta: enero de 2017].

Oliveira, Ismar (2010), "Caminos de la educomunicación: utopías, confrontaciones, reconocimientos", en Educomunicación: más allá del 2.0, Barcelona, Gedisa, pp. 129-150. 
Olmedo, Silvia e Isabel María Ruiz (2013), "Consolidar, una agencia de comunicación solidaria para las ONGD en la universidad", en Buenas prácticas de colaboración entre ONGD y universidad: educar en la ciudadanía global en el espacio universitario, Córdoba, Fundación ETEA, pp. 105-111.

Pérez-Reina, Sira Silvia (2011), "Relación de las Universidades con su Entorno desde la Prospectiva de la Responsabilidad Social Universitaria", en Revista Digital de Investigación y Postgrado de la Universidad
Nacional Experimental Politécnica "Antonio José de Sucre", Vicerrectorado Barquisimeto, Venezuela, vol. 1, núm. 3, pp. 191-209.

Restrepo-Salazar, María Eugenia, Jiménez-Dalorzo, Leidy y Nancy Sánchez-Acuña (2015), “Extensión universitaria y prácticas estudiantiles: una experiencia de formación curricular en la comunidad", en Revista Universidad en Diálogo, vol. V, núm. 1, pp. 111-129.

\section{Cómo citar este artículo:}

Rascón-Gómez María-Teresa, Florencio Cabello Fernández-Delgado y Alejandro Alvarado-Jódar (2019), "Miradas multimedia sobre resiliencia y educación: innovación educomunicativa para la resiliencia de la infancia en riesgo social", en Revista Iberoamericana de Educación Superior (RIES), México, UNAM-IISUE/Universia, vol. X, Núm. 28, pp. 157-169, DOI: https://doi.org/10.22201/ iisue.20072872e.2019.28.434 [consulta: fecha de última consulta]. 